GESTIÓN DEL CLIENTE-PACIENTE ODONTOLÓGICO COMO DIMENSIÓN DEL CAPITAL RELACIONAL.

\title{
Gestión del Cliente-Paciente Odontológico como Dimensión del Capital Relacional
}

\section{Dental Client-Patient Management as a Dimension of Relational Capital}

\author{
Herrera Ángela*, Soto Aguirre, Neirys**
}

\begin{abstract}
*Doctorando del Doctorado en Ciencias Administrativas y Gerenciales (FaCES-UC). Universidad de Carabobo (UC), Venezuela. Profesora Instructor a tiempo cumplido (Facultad de Odontología-UC). Especialista en Ortodoncia (Bauru, SP- Brasil). Magister Scientarum en Gerencia de Salud Pública (UNERG). Email: dra.angela.herrera@gmail.com, ORCID: https://orcid.org/0000-0002-8338-1552.

**Doctora en Ciencias Económicas y Sociales. Universidad de Carabobo (UC), Venezuela. Profesora Asociado Dedicación Exclusiva de la Facultad de Ciencias Económicas y Sociales (FaCES-UC). Investigadora del Centro de Investigación de Sociedad, Economía y Transcomplejidad (CISET-FaCESUC). Investigador Nacional PEII-ONTIC. Email: neiso05@gmail.com, ORCID: https://orcid.org/0000-00028942-7304.
\end{abstract}

Correo para recibir correspondencia: nsoto1@uc.edu.ve.com

Fecha de recibido: 18 de marzo de 2021

Fecha de aceptación: 11 de junio de 2021 

RELACIONAL.

\section{RESUMEN}

OBJETIVO: Comprender la gestión del cliente-paciente dentro de la gerencia de las organizaciones odontológicas con base en la dimensión del capital relacional.

MATERIAL Y MÉTODO: Es una investigación de tipo documental y emplea los métodos descriptivo y analítico; esto implicó la determinación de los componentes, elementos y estrategias que activan la dimensión relacional, para luego identificar al cliente-paciente odontológico dentro de la naturaleza del ecosistema organizacional y su comportamiento como entidad lingüística que acciona la búsqueda de su propio mejoramiento, cambio y desarrollo.

RESULTADOS: Obteniéndose como hallazgo, tanto la significación del cliente-paciente odontológico como la interpretación de los elementos del capital relacional dentro de su forma de vida fundamentado en reconfiguraciones lingüísticas que generan los modos de actuar.

CONCLUSIONES: La dimensión cliente-paciente odontológico gira entorno a la identidad individual o colectiva que desea con respecto a su sonrisa, lo cual va más allá de sanar una dolencia, esto demanda de un ámbito odontológico revolucionado bajo un paradigma estético soportado en la Armonización Orofacial, asimismo, el odontólogo requiere competencias gerenciales que les permita gestionar tanto las expectativas como las necesidades que buscan los clientes-pacientes fundamentado en el capital relacional con base en la marca, la identidad, la reputación e imagen corporativa del profesional u organización odontológica.

PALABRAS CLAVE: Capital relacional. Gestión. Odontología. Cliente-paciente odontológico.

\section{ABSTRACT}

OBJECTIVE: To understand the client-patient management within the management of dental organizations based on the dimension of relational capital.

MATERIAL AND METHOD: It is a documentary type investigation that uses descriptive and analytical methods; This implied the determination of the components, elements and strategies that activate the relational dimension, to later identify the dental client-patient within the nature of 

RELACIONAL.

the organizational ecosystem and its behavior as a linguistic entity that triggers the search for their own improvement, change, and development.

RESULTS: obtained as a finding, both the significance of the dental client-patient and the interpretation of the elements of relational capital within their way of life-based on linguistic reconfigurations that generate the ways of acting.

CONCLUSIONS: The dental client-patient dimension revolves around the individual or collective identity that they want with respect to their smile, which goes beyond healing an ailment, this demands a revolutionized dental field under an aesthetic paradigm supported by Orofacial Harmonization. Likewise, the dentist requires managerial skills that allow him/her to manage both the expectations and the needs sought by clients-patients based on relational capital based on the brand, identity, reputation, and corporate image of the dental professional or organization.

KEYWORDS: Relational capital. Management. Odontology. Dental client-patient.

\section{INTRODUCCIÓN}

Si hacemos una retrospectiva, la economía de la era industrial precisaba a los elementos tangibles como las fuentes absolutas de valor; ahora bien, esta nueva era de la información, se soporta en el conocimiento, en la creación y avance de las comunicaciones, en las redes multimedia, la robótica, la nanotecnología, entre otros; de hecho, estas son las estructuras mediante las cuales las organizaciones empresariales gestionan los beneficios futuros, constituidas por los elementos intangibles denominados capital intelectual, el cual representa según Bontis (1998) un constructo multidimensional conformado por tres dimensiones: capital humano, capital estructural y capital relacional. Al respecto Merino \& Díaz (2008) explican que el valor resultante del conjunto de intangibles puede determinar tanto el presente como el futuro de la organización, en este sentido, el reto del gerente actual "yace en la postura a seguir para lograr capitalizar los recursos intangibles disponibles" (Cortina, Flores \& Álvarez, 2019; FloresFlores, Bernal-González \& Valentín-Mballa, 2020). 

RELACIONAL.

En este momento investigativo, se realizará una reflexión acerca de la necesidad de entender como la gestión del cliente-paciente influye en la función gerencial para el direccionamiento de las organizaciones odontológicas, teniendo como eje temático medular el capital relacional, el cual explican Edvinsson \& Malone (1999) que consta, principalmente, por el valor generado por las relaciones de la empresa con sus clientes, considerando, que según Flores, Verástegui \& Hernández (2021) "las relaciones a largo plazo con los usuarios, así como alianzas estratégicas, son los factores que mayor nivel de asociación estadística tienen sobre [...] el desarrollo organizacional" (p. 13). Para darle profundidad epistémica a la dimensión relacional, se desarrollarán tanto sus componentes como los elementos que lo activan hasta precisar la gestión del cliente, con énfasis particular en el cliente-paciente odontológico como entidad lingüística dentro de esta dinámica empresarial.

\section{MATERIAL Y MÉTODO}

Se utilizará un estudio descriptivo mediante un análisis de contenido con el fin de caracterizar la gestión del cliente-paciente odontológico, para ello esgrimimos los elementos fundamentales del capital relacional dentro de las empresas, deteniéndonos en el análisis organizacional en el ecosistema odontológico.

\section{MARCO EPISTÉMICO}

Muchos autores han contribuido al desarrollo conceptual del capital intelectual entre los que se destacan Bontis (1996, 1998, 1999, 2000), Roos, Roos, Dragonetti y Edvinsson (1997), Stewart (1991, 1994, 1997), Sveiby (2000), Edvinsson y Malone (1997), Saint-Onge (1996), Sullivan y Edvinsson (1996), Bueno (1998, 2008), Chiavenato (2011), Aramburu, Sáenz \& Blanco (2015), ellos enfatizan que este debe ser conceptualizado como la adición de tres componentes básicos: capital humano, capital estructural y capital relacional, dado que, la combinación de estos activos inmateriales o intangibles crean valor futuro y sobre los cuales se puede sustentar una ventaja competitiva sostenida (Sanchez, Melia \& Garcia, 2007).

En efecto, las empresas de este nuevo entorno competitivo deberán utilizar una inteligente estrategia de gestión de sus activos intangibles (Borrás-Atiénzar \& Campos-Chaurero, 2018), que le permita concebir a la organización como un sistema abierto, aprendiendo del 

RELACIONAL.

ecosistema, de integrar a los clientes, de estrechar lazos en las relaciones, del buen uso de la información y de saber aprovechar el talento.

En este sentido, Hsu \& Fang (2009) demuestran la pertinencia en el aprendizaje organizacional, donde lo más importante radica en el capital relacional, puesto que, esta dimensión incluye las relaciones con el entorno, específicamente con los agentes que participan en la cadena de valor, los cuales generan las relaciones de la empresa, no sólo con clientes, proveedores y accionistas, sino con todos sus grupos de interés, tanto internos como externos (Bontis, 1996; De Pablos (2003); Stewart (1998); Roos, Roos \& Dragonetti (2001). Podemos conceptualizarlo como el conocimiento que se encuentra incluido en las relaciones de la organización (Bontis, 1998,1999; Sanchez, Melia \& Garcia, 2007). Cabe decir que, son vínculos que se establecen con el medio, sobre todo con los clientes a los cuales se les debe cumplir las expectativas con mayor eficiencia en base a la calidad de servicio; sin dejar de lado, los proveedores, las alianzas estratégicas con diversas instituciones lo cual nos brindara una mejor gestión.

\section{Entendiendo el capital relacional}

Este capital se puede conceptualizar como el conjunto de conocimientos basado en las relaciones institucionales que mantiene una empresa con otros agentes, que le reporta un valor y una base de conocimientos necesarios para realizar su actividad de manera más eficiente (Delgado-verde, Martin-de-Castro, Navas-López, \& Cruz- González, 2011). De forma que, "equivale a todas las redes formales de colaboración creadas por la organización para alcanzar, de la manera más eficiente posible, los diversos objetivos y metas previamente establecidos durante la etapa de planeación estratégica" (Flores, Verástegui \& Hernández, 2021, p. 3). Es decir, que se define como el acumulado de todas las relaciones que se constituyen entre la sinergia de organizaciones, personas y corporaciones que tienen una capacidad de cooperación con la empresa.

Es evidente entonces, que la calidad de las relaciones en las organizaciones caracterizadas por la transparencia y la confianza obtiene ventajas importantes, en relación con las estrictamente puntuales con el entorno. Desde el punto de vista de Sanchez, Melia \& Garcia (2007) el capital relacional de las empresas no son sistemas aislados, sino que por el contrario, se vinculan con el exterior, cabe decir que, la dinámica de las personas que se relacionan entre sí, consolidan la organización. Sobre la base de las consideraciones anteriores, los gerentes deben examinar las 

RELACIONAL.

posibilidades internas y externas; siendo de esta manera, la empresa que nutre al capital relacional contando con la destreza de identificar e interactuar positivamente con los diferentes actores claves de su entorno (Diaz, 2007).

Las dimensiones fundamentales que engloba el capital relacional constituyen la base para la obtención de ventajas competitivas, para los autores Edvisson \& Malonne (1999) el capital relacional (capital-cliente) es una categoría separada, equivalente a capital estructural y humano, sugieren que las relaciones de una empresa con sus clientes son distintas de su trato con los empleados y los socios estratégicos; de hecho, este capital apunta hacia unos índices de medición que incluyen satisfacción, longevidad, sensibilidad y bienestar financiero, los cuales deben ser fácilmente comprensibles, aplicables y comparables entre distintas organizaciones. Es evidente entonces, que las relaciones de la empresa están vinculadas con agentes externos, y depende fundamentalmente de la capacidad de sus miembros.

Entre tanto, Stewart (1999) visualiza al capital relacional de una manera particular, como el más axiomático de los tres tipos del capital intelectual, reconoce que gracias a los clientes existe la empresa y que es un activo que crea valor. En este propósito, la inversión en ellos podrá generar una retroalimentación que evitara errores. Otra aportación teórica importante al respecto es la señalada por Bontis (2001) quien propone que el conocimiento de los canales de marketing y las relaciones con los clientes es significativo en el capital relacional, representan el potencial que tiene una empresa a causa de los intangibles que contienen el conocimiento asociado en clientes, proveedores, el gobierno o asociaciones industriales; este autor considera que esta dimensión es la más externa al núcleo de la organización, y la más valiosa a medida que pasa el tiempo.

\section{Componentes del capital relacional}

Según Bueno, Salmador \& Merino (2008) este capital constituye el arte de interacción empresarial con el ecosistema que confluyen generación de riqueza, el cual contempla dos tipos: el capital relacional de negocio y el capital relacional social; el primero se refiere a los flujos de información y conocimiento de carácter externo vinculados al negocio (proveedores, clientes-usuarios, aliados, competidores, medios de comunicación, imagen corporativa, instituciones de promoción y mejora de la calidad); mientras el segundo, le concierne al marco de relaciones fuera del ámbito del negocio en el cual se integran las relaciones con accionistas 

RELACIONAL.

e inversores, con administraciones públicas, con la defensa del medio ambiente, las relaciones sociales, la reputación corporativa y otras relaciones con la sociedad (Flores, Verástegui, \& Hernández, 2021).

Siguiendo la misma línea Bueno, Del Real, Fernández, Longo, Merino, \& Murcia, (2011) puntualizan un modelo de capital relacional en las organizaciones alrededor de sus principales agentes: i) relaciones con clientes; ii) relaciones con aliados; iii) relaciones con proveedores; iv) relaciones con accionistas e inversores; v) relaciones con otros agentes; vi) reputación de la empresa, etc. Destacando que, Bueno, et al., (2011) proponen que el agente (i) constituye el eslabón para obtener el éxito competitivo empresarial.

\section{Elementos que activan el capital relacional}

Las grandes empresas para permanecer en la dinámica actual de los negocios utilizan varios de los elementos que favorecen la dinamización del capital relacional mencionados anteriormente, tales como: marca, identidad e imagen corporativa, reputación corporativa, relaciones informales y familia; ellos son naturaleza intangible que destacan la diferencia.

\section{- La marca}

Representa un símbolo para la identificación, toma de decisiones de consumo e intercambio, por lo que se han logrado convertir hoy día, en ejes principales sobre la cual se soporta los comercios, adquiriendo valor y ubicándose como protagonistas en el mundo de la publicidad y el mercadeo; influenciando no solo en las decisiones de compra y venta de productos, sino además los estilos de vida, las relaciones, los intereses ambientales, económicos, políticos y hasta religiosos (Cepeda-Palacio, 2014). En el pasado, Philip kotler se refiere a las marcas desde la significación propuesta por la American Marketing Association, la cual las define como "nombre, termino, signo, símbolo o diseño o combinación de ellos, cuyo objeto es identificar los bienes o servicios de un vendedor o grupo de vendedores con objeto de diferenciarlo de sus competidores" (Kotler, 2000, p. 487).

Sin embargo, tiene ciertas limitaciones que no permiten develar los alcances, funciones y realidades de lo que es la marca en el contexto contemporáneo; ya que se debe entender su evolución; es decir, los diferentes caminos que han transitado hasta llegar al mundo actual. Para empezar, la percepción de la marca varía de individuo a individuo, como si la misma tiene 

RELACIONAL.

su propio significado, por lo tanto, al conocer debidamente sus alcances en la sociedad actual se hace posible entender la forma en que está concebida y evitar tener interpretaciones de manera subjetiva. De aquí, la importancia de entender el fondo de lo que significa la marca (Cepeda-Palacio, 2014).

Así, las marcas se vincularon con mayor fuerza al sistema de producción capitalista, durante el apogeo de la era industrial del siglo XIX, por la cual eran utilizadas originalmente por las campañas publicitarias para promover las nuevas invenciones que salían al mercado, buscaban más la adaptación de las personas a estos nuevos consumos y a los cambios en el estilo de vida que estos implicaban. Mencionando la escritora Naomi Klein (2001) "Los publicitarios enfrentaban tareas más urgentes que la de crear marcas que identificaran a las empresas; primero tenían que cambiar la manera en la que la gente vivía sus vidas" (p. 33), lo que quiere decir, que los anuncios se centraban en enunciar las bondades de los nuevos productos y la forma en que su uso podía simplificar o mejorar el estilo de vida de las personas.

Como consecuencia de esto, y detrás de la evolución de los signos representativos, se hace posible demostrar la ampliación en su concepto con relación a sus alcances, lo que quiere decir, que actualmente estas pueden representar una empresa, un producto o servicio, una emoción, un individuo e inclusive un accionar. Siendo un punto de convergencia el factor identificación, el cual permite distinguir una marca de otra, como lo plantea Boone \& Kurtz (2012) "un logotipo de la marca es un símbolo o diseño gráfico que distingue a un producto" (p. 385). No obstante, se expone una definición basada en la concepción técnica y tradicional de los signos representativos: "las marcas son iniciativas completamente basadas en el diseño e integradas de una forma sistémica en los esfuerzos del marketing" (Atkin, 2008, p. 135), comprobándose para otros expertos que la marca es un factor de protección no solo de los productos, sino también de las empresas a las que esta representa.

\section{- La identidad y la imagen corporativa}

La gestión de la identidad y la imagen de la empresa han adquirido relevancia notable para las corporaciones modernas, lo que hace que se obtengan ventajas competitivas. Entonces, la identidad corporativa se proyecta con el objetivo de generar una impresión (imagen) especifica en los stakeholders con los que se relaciona la empresa; sin embargo, cerca de la noción de identidad han aparecido un conjunto de definiciones interrelacionados como identidad 

RELACIONAL.

corporativa, identidad de marca, identidad organizacional, identidad visual o identidad de negocio (Currás, 2010). En relación a esto, Balmer (2001) emplea la identidad de negocio para relacionar la identidad visual, la organizacional y la corporativa, donde la visual hace referencia a la representación gráfica de la corporativa en forma de símbolos y señales (Chajet \& Shachtman, 1998; Riel, 1995).

Desde la perspectiva más general, Margulies (1977) define la identidad corporativa como todos los mecanismos que una empresa elige para identificarse ante sus stakeholders (la comunidad, clientes, trabajadores y medios). Sin embargo, (Balmer, 2001) expresa que es la suma de los elementos tangibles e intangibles que distinguen a una organización y que se encuentra ordenada por la acción de los líderes, por la tradición y el entorno de la organización; a su vez, se muestra a través de múltiples canales, todo esto apuntando al área de estudio en la que se situé el investigador. Cabe agregar, que según Currás (2010) la identidad es el mecanismo de representación de lo que es la empresa realizando énfasis en los medios simbólicos (logos e identidad visual destacadamente).

En ese sentido, la identidad corporativa es un sistema de comunicación que se agrega a la estrategia integral de la organización y es el proceso transformador a la imagen. Es por esto, que gestionar la identidad es gestionar la imagen, más no viceversa. Por lo cual, la misma posee una interdependencia con la identidad de una organización. En relación a esto, Terkla \& Pagano (1993) opinan que son un conjunto de percepciones individuales acerca de la institución. Sin embargo, para Martineau (1958) el termino Imagen es la suma de las cualidades funcionales y atributos psicológicos que existen en la mente del consumidor. Para Costa (1977) la imagen de la empresa es la resultante de múltiples y diversos mensajes que, acumulados en la memoria colectiva, configuran una globalidad significativa capaz de influir en los comportamientos y determinarlos. Pero, para impedir caer en definiciones y aproximaciones improductivas para el campo de la administración, el enfoque se dirige a la imagen corporativa, que con el paso del tiempo ha adquirido notabilidad dentro de la planeación estratégica de las organizaciones e incluso un componente dentro de las empresas (Duque \& Carvajal, 2015).

En tal sentido, Villafañe (1998) conceptualiza a la imagen corporativa como el resultado de la integración, en la mente del público con los que la empresa se relaciona, de un conjunto de imágenes que, con mayor o menor protagonismo, la empresa proyecta hacia el exterior. Asimismo, Christie (2002) puntualiza la imagen corporativa con un término holístico que incluye 

RELACIONAL.

tres perspectivas interrelacionadas: la autoimagen, la imagen proyectada y la imagen percibida de la organización. Por lo tanto, se ha establecido entre estos términos una relación sobre todo en los estudios provenientes del área del marketing, los cuales han sido basados en un modelo comunicativo clásico emisor-receptor, donde la imagen depende de la identidad (Christensen y Askegaard, 2001; Dowling, 1986; Margulies, 1977). Dejando en claro, Hatch y Schultz (1997) que la interdependencia de la relación identidad e imagen cumple la cultura organizacional, cabe decir que, son la clave para que las empresas se puedan posicionar y diferenciar de su competencia ya que la identidad corporativa es un factor interno que debe puntualizar la empresa para generar una impresión específica en sus clientes potenciales, mientras que la imagen corporativa es como se percibe la marca.

\section{- La reputación}

Es como un activo de construcción social que es instaurada y sostenida por los procesos de legitimación (Rao, 1994), lo que involucra una cantidad de evaluaciones de las organizaciones de manera sucesiva basándose en los criterios de los mismos, actuando dentro del marco institucional; entonces, se entiende la legitimidad como un precedente a la reputación siendo así una variable de flujo. A su vez, está vigorosamente relacionada con otros procedentes de diferentes campos de la dirección de empresas, principalmente del área comercial y de marketing, en especial, con la imagen e identidad corporativa (Gotsi \& Wilson, 2001). La reputación es un activo intangible que está relacionado con la organización, que consiste en los juicios y valores que se obtiene sobre la imagen de la empresa a través de clientes, competidores, empleados potenciales y stakeholders (Michalisin, Kline, \& Smith (2000); Rodriguez (2004). Entonces, es un elemento que produce la seguridad al sector y a los clientes siendo la respuesta de la interacción del prestigio o renombre que pueda preceder al empresario, generando ayuda para adquirir nuevos clientes y fidelizar los actuales.

\section{- Las relaciones informales}

Han sido una evolución en el capital relacional, representan las interacciones entre las personas en un contexto de cercanía, de amistad o de sintonía fraccionando las barreras estructurales organizativas. En las mismas, existen canales de comunicación a través de los cuales fluyen contenidos que no pueden ser estudiados aisladamente, sino que se interrelacionan, se expanden, se contraen y tienen movimiento predecible (Brûderl \& 

RELACIONAL.

Preisendorfer, 1998). En otras palabras, los contactos o redes de relaciones pueden brindar información, recomendar, ofrecer una ocupación, proporcionar prestamos económicos, lo cual contribuyen en la gestión de la organización (Carrascosa, 2021).

\section{- La familia}

Generalmente brinda un apoyo emocional; entre estos, están los familiares y amigos, que se convierten en una importante fuente de financiación en muchas oportunidades, además de mano de obra lo cual hace un ahorro significativo en el gasto personal al comienzo de los primeros años de vida de la organización. Por otra parte, la fidelidad y la seguridad que se obtiene con los miembros de la familia laborando dentro del negocio u organización suponen un menor esfuerzo en la tarea de control de la actividad (Brûderl \& Preisendôrfer, 1998), adicionalmente, el apoyo de la pareja puede contribuir un valor de la empresa, aportándole estabilidad emocional.

De todo esto se desprende que, la suma de estos elementos son los que sobrellevan al capital relacional colocando de manifiesto que el emprendedor recibe tanto apoyo emocional como estructural de sus relaciones más cercanas, generándose un valor para la organización que tiene una relación positiva con el éxito, de forma que, se genera un impacto directo teniendo influencia en sus resultados. Es por esto, que gestionar los clientes requiere disponer de esos elementos para anticiparse a las necesidades de estos, y fomentando el manejo del capital relacional con ellos, en este sentido, Miranda, Chamorro \& Rubio (2007) plantean que una buena gestión se basa en el compromiso, en las normas orientadas hacia el cliente, en la formación, la delegación y la efectividad de la gestión. De modo que, Barquero (2008) asegura que la gestión de clientes tiene un resultado multiplicador dentro del mercado por el elemento comunicación. Así mismo, consideran que para cimentar la relación deben añadir beneficios financieros, sociales y de dependencias estructurales (Lorenzo \& Moreira, 2009).

\section{Descripción del ecosistema organizacional odontológico para comprender la interacción con el cliente-paciente}

Haciendo un poco de historia la odontología tuvo sus primeros registros arqueológicos aproximadamente desde hace unos 14 milenios, en la era antigua 200 A.C., los egipcios fueron los primeros curanderos que trataban los problemas dentales, también incrustaban piedras 

RELACIONAL.

preciosas en los dientes como adorno a los grandes faraones e incluso realizaban extracción dentaria, puesto que, era la forma principal de aliviar este tipo de afecciones; tres siglos más tarde, en el continente Asiático se utilizó la acupuntura para aliviar el dolor relacionado con caries. (Leal \& Hernández, 2016).

Es conveniente acotar que, la odontología ocupa un lugar exclusivo en el organismo de las Naciones Unidas que se fundó el 24 de octubre de 1945; así como también en la Organización Mundial de la Salud (OMS) la cual entró en vigor el 7 de abril del año 1948; actualmente, son más de 7000 personas trabajando en 150 oficinas de país, seis oficinas regionales y la Sede de Ginebra, la OMS (1948), y su Oficina Regional para las Américas, la federación dental internacional más antigua del mundo, que se fundó en Francia en el año 1900 por iniciativa del odontólogo francés Charles Godon; y es la voz mundial, autorizada e independiente de la profesión dental FDI (1900).

En el caso específico de Venezuela, la Ley de ejercicio de la odontología en su Capítulo I, Artículo 2, (1970) la define como:

La prestación de servicios encaminados a la prevención, diagnóstico y tratamiento de las enfermedades, deformaciones y accidentes traumáticos de la boca y de los órganos o regiones anatómicas que la limitan o comprenden. Tales intervenciones constituyen actos propios de los profesionales legalmente autorizados, quienes podrán delegar en sus auxiliares aquellas intervenciones claramente determinadas en esta Ley su Reglamento (p.1).

Asimismo, la odontología se conceptualiza como una carrera del área de la salud, donde se proyecta de manera activa la teoría de los procedimientos, dirigidos hacia la mantención y recuperación de la salud bucal humana en una atención de calidad hacia el paciente (Sanhueza \& Cabrera, 2014). Cabe destacar, también, que la odontología es una profesión de servicio social en el campo de la salud bucal (Beltrán, 2013). De hecho, es una labor con identidad propia, su ejercicio profesional es independiente y su capacidad es transdisciplinaria; siendo, su campo de acción toda la cavidad oral como maxilares, músculos, piel, vasos y nervios. En otras palabras, es una especialidad médica que se dedica al estudio de los dientes, encías y el aparato estomatognático que está formado por un conjunto de órganos, tejidos en la cavidad oral y parte de cráneo, cara y cuello. 

RELACIONAL.

En el siglo XXI, la praxis odontológica incluye teorías innovadoras dentro del diagnóstico, implantes osteointegrados, aparatología ultrasónica, odontología digitalizada, e incluso la especialidad de Armonización Orofacial aprobada hoy en día en Brasil y Venezuela; lo que hace que el profesional pueda sumergirse en un mundo lleno de posibilidades con un amplio espectro de información para producir más satisfacción y cubrir las expectativas a sus clientespacientes. Con la aparición de la epidemia del SARS (síndrome respiratorio agudo severo), en china se utilizaron iniciativas ingeniosas que condujeron al progreso en la educación médica. Se implementaron técnicas de aprendizaje en línea los cuales resultaron populares y son aplicados hasta la actualidad de forma complementaria (Márquez 2020, QuispeJuli \& et al.).

Siendo, la telesalud el conjunto de actividades que se llevan a cabo a distancia, en las áreas de promoción, prevención, diagnóstico, tratamiento o rehabilitación; por profesionales de la salud que manejan tecnologías de información y comunicación, la cual ha tomado protagonismo, debido a que los centros de salud podrían ser fuentes de contagio. "Aplicando especialmente la atención a través de video llamada o teleconsulta, lo que evita el riesgo de contagio y reduce así la propagación del COVID-19" (Almazrooaa et.al. 2020). Por ende, estos sistemas de telemedicina se desarrollan para la computación generalizada y una de sus subcategorías es la teleodontología, con la que es posible realizar consultas distantes, compartiendo información digital como imágenes, trabajo cooperativo, documentos, radiografías, entre otros (Márquez, 2020).

La Association American Teledentistry (ATDA) puntualiza la teleodontología como:

"El uso de tecnologías electrónicas de información, imagen y comunicación, que incluyen comunicaciones interactivas de audio, video y datos, así como tecnologías de almacenamiento y reenvío, para proporcionar y apoyar la prestación, el diagnóstico, la consulta, la transferencia de información dental y la educación sobre la atención dental (Association American Teledentistry, 2019).

Esta puede incluir las siguientes modalidades: el video en vivo (síncrono); el almacenamiento y reenvió (asíncrono); el monitoreo remoto de pacientes (RPM) y la salud móvil respaldada por dispositivos de comunicación móvil y aplicaciones de software, incluidos teléfonos celulares, tabletas y asistentes digitales personales (Araujo \& Morón, 2021).

Cabe destacar, que la teleodontología es un apoyo al sistema de salud que permite agilizar de manera selectiva la asistencia clínica para la práctica odontológica, lo que hace que sea 

RELACIONAL.

fundamental que el paciente consiga un espacio que más que atractivo, tenga las normas de bioseguridad y, a su vez, disponga de la operatividad necesaria para desempeñar el trabajo de la forma más óptima; teniendo como criterios claves: funcionalidad, comodidad, accesibilidad seguridad y privacidad.

La organización odontológica es de naturaleza lucrativa o no lucrativa, privada o pública, mixta, cooperativas, entre otras; cuya actividad medular se basa en la provisión de servicios "en este sentido, podríamos decir que la finalidad organizacional no siempre posee finitud y, en los casos en que sí pueda aplicarse este criterio de finitud, la finalidad reside en la existencia 0 inexistencia de una necesidad social no satisfecha" (Marcó, Loguzzo \& Fedi, 2016), en este caso en particular, el fin se concibe en la prevención, promoción, diagnóstico y tratamiento de atención de la salud bucal, cuyo accionar organizacional se fundamenta en los pilares de la administración: planeación, organización, dirección y control, con el propósito de alcanzar la eficacia y la eficiencia las cuales representan dos dimensiones del desempeño de una empresa.

\section{Identificación del cliente-paciente odontológico}

Sobre la base de las consideraciones anteriores, se desprende que las relaciones con los clientes son las que conservan las ventajas competitivas y de carácter de hermandad, por lo tanto, deben estar alineadas con el entorno y hacerse más fuerte para posicionarse cada día más, aquí en particular, la reflexión se inscribe en el cliente-paciente odontológico como fundamento en el direccionamiento de las organizaciones odontológicas.

Partiendo de la noción de paciente que remite a una relación intersubjetiva fundada en una serie de principios éticos concebidos para neutralizar la asimetría de poder, y cuyo sentido se consuma en el ejercicio de la compasión ante el dolor del sujeto enfermo; para otros autores, el paciente es un ente pasivo, es un objeto en manos del médico como mecánico que se esfuerza por componer las funciones alteradas (Sala, De Cárdenas, Sala \& González, 2000); en otras palabras, un individuo que es examinado o se le administra tratamiento. No obstante "un cliente es toda persona, grupo o entidad, que recibe servicios de otra, independientemente de que pague o no" (Núñez, 2000, p. 113); es decir, es la persona, empresa u organización que adquiere de manera consciente productos o servicios que precisa o desea. De hecho, el cliente es la razón por la cual la organización investiga, planifica, implementa y controla todas sus actividades para mantener la satisfacción y la fidelización (De la Hoz, 2017). En este sentido, se 

RELACIONAL.

puede con ello significar al cliente-paciente odontológico como la persona usuaria que solicita y recibe servicios de salud bucal que contempla desde el diagnóstico, el tratamiento y la prevención de las enfermedades del aparato estomatognático para acomodar las funciones alteradas, y cuya clave para el buen manejo de la relación con él, está en la capacidad que tenga el odontólogo para gestionar sus necesidades y expectativas con respecto al producto 0 servicio que se le ofrece.

Para entender mejor las particularidades de la gestión del cliente-paciente odontológico es necesario razonar que la relación médico-paciente depende de los criterios bioéticos y legales, además de la concepción de la autonomía de los pacientes. Por otra parte, la revolución tecnológica, científica, social, cultural, económica y política han impulsado a cambios, lo cual ha generado la adquisición de habilidades comunicativas siendo la entrevista médica la herramienta fundamental para obtener una anamnesis fidedigna, la cual posee valor terapéutico cuando el cliente-paciente odontológico encuentra en el profesional capacidades de respeto, interés, autenticidad y confianza. Para los odontólogos, igual que para los médicos es de vital importancia ganarse la confianza de los clientes-pacientes, para que los mismos sean honestos y cooperen en el interrogatorio, de lo contrario, en la historia clínica negaran cualquier situación sistémica que pueda comprometer su condición general de salud ya que gran parte de estos consideran que los órganos dentarios son estructuras separadas del resto del cuerpo (Mancera, 2021). Por lo tanto, los cambios en el área de la odontología han creado nuevos paradigmas en la relación odontólogo-paciente iniciando un modelo donde se exige al profesional informar todo lo relacionado con su salud bucal; utilizando el consentimiento informado como un instrumento escrito que pone de manifiesto las voluntades expresadas: el derecho del cliente-paciente a la elección responsable y el respeto a la propia libertad sobre su salud, focalizándose en la práctica clínica, la ética y la moral en los consultorios odontológicos (Damián-Navarro, FloresMori y Flores-Mena, 2014).

Por consiguiente, el odontólogo ya no es un ejecutor supremo, no debe asumir unilateral e individualmente los riesgos inherentes, debe compartir todas las informaciones relacionadas al proceso de la atención clínica con el mismo (Maluf, Santos de Melo \& Garrafa, 2014). De modo que, en la práctica odontológica el respeto hacia la autonomía antes descrita es reconocer que el cliente-paciente tiene derecho a tomar decisiones cimentado en valores propios, expectativas y aspiraciones personales. 

RELACIONAL.

En síntesis, la comprensión del vínculo odontólogo-paciente basado en la integración del saber profesional y rol del cliente-paciente odontológico tiene implicaciones en una realidad centradas en el mejoramiento de la condición humana. La asistencia profesional en la odontología es más que un experto al lado del sillón, se conforma de una estructura y un vínculo desde la consulta inicial hasta el proceso terapéutico donde interviene el ámbito afectivo, cognitivo, operativo y moral (Saenz, 2013).

\section{El cliente-paciente como entidad lingüística dentro de la organización odontológica}

A partir de la necesidad de gestionar al cliente-paciente odontológico se hace imperativo comprender al ser humano como entidad lingüística, puesto que, provee de sentido su forma de vida fundamentado en reconfiguraciones lingüísticas que generan los modos de actuar, en este sentido, el lenguaje revoluciona al centrarse como paradigma. Al respecto, Echeverría (2009) plantea que las personas interpretan el mundo desde "su mirada, su atención, sus omisiones, sus recuerdos, sus asociaciones, su emocionalidad" (p. 96); es decir, desde la complejidad de sus mundos, de hecho para Heidegger (1972), citado por Echeverría (2009) "no podemos separar el ser que somos, del mundo dentro del cual somos" (p. 96); de allí, que el hombre y mundo existe de manera paralela y referencial.

Ahora bien, existen dominios primarios que delimitan la interpretación de cada ser humano estos son "la corporalidad, la emocionalidad y el lenguaje" (ídem), dicho dominios definen lo que es y quiere ser la persona en su mundo interpretativo con referencia a lo que le rodea. Esa interpretación confluirá en la díada inquietud /deseo la cual contempla "diversos juicios y emociones que dan cuenta de que aquello que sucede, nos importa, nos atañe, de determinadas maneras" (Echeverría, 2009, p. 103); en suma, la díada conferirá el accionar del individuo en su búsqueda de su propio mejoramiento, cambio y desarrollo.

Es conveniente acotar que, también nos encontramos inmerso en una sociedad de consumidores que según Bauman (2007) "interpela a sus miembros (vale decir, se dirige a ellos, los llama, los convoca, apela a ellos, los cuestiona, pero también los interrumpe e irrumpe en ellos) fundamentalmente en cuanto a su capacidad como consumidores. Al hacerlo de este modo, la sociedad (cualesquiera sean los mecanismos humanos muñidos de las armas de coerción y los medios de persuasión que se oculten detrás de ese concepto o imagen) espera ser escuchada, atendida y obedecida" (p. 77). 

RELACIONAL.

De lo anteriormente señalado, se puede observar que nos encontramos en una realidad soportado en un sistema que cada día genera cúmulos de expectativas basadas en arquetipos sociales y estándares de belleza compartidos por un grupo de interés, que basados en el lenguaje construyen nuevas necesidades, objetos, productos y realidades (Echeverría, 2000); esto genera en las personas aspiraciones de encajar, de allí, surgen inquietudes y deseos, que los impulsa en la búsqueda de organizaciones como las odontológicas que puedan alcanzar las competencias de mejora o cambio de la belleza facial y dental soportada en la comunicación necesaria para garantizar el éxito a largo plazo.

Por lo tanto, los clientes-pacientes odontológicos acuden a una consulta por una parte, cuando tienen dolor o molestia para sanar y por la otra, con el deseo de alcanzar la identidad individual o colectiva que desean con respecto a su sonrisa, lo cual va más allá de restauraciones bucales, girando la medicina odontológica desde la estética dental hacia nuevas tendencias como la Armonización Orofacial, la cual es una especialidad odontológica que busca la rehabilitación funcional y estética del sistema estomatognático, buscando mediante sus procedimientos equilibrio, función, estética de los dientes y de la cara, asociando áreas como la implantología con laserterapia, toxina botulínica y ácido hialurónico, entre otros (Souza \& et.al, 2021). En este sentido, esta soportada en las estructuras adyacentes (labios, mejillas, mentón, entre otros), motivada principalmente, por las nuevas significaciones en los clientes-pacientes odontológicos que desean estar saludables, que aspiran impactar con una imagen impecable y unido al advenimiento de nuevos materiales, donde además se incluye la innovación y la tecnología, que satisfacen con efectos inmediatos los requerimientos de belleza, funcionalidad y de rejuvenecimiento facial.

\section{CONCLUSIONES}

La dimensión cliente-paciente odontológico va más allá de sanar una dolencia, puesto que, hoy día el ser humano se fundamenta en las configuraciones del mundo lingüístico donde sus inquietudes y deseos son dinamizados por su mundo simbólico de referencia que gira en torno a la belleza, lo que demanda de un ámbito odontológico revolucionado bajo un paradigma estético, con la aparición de la armonización orofacial lo cual le puede proporcionar al clientepaciente odontológico esas necesidades intrínsecas (reconfiguraciones lingüísticas) que definen su forma de actuar y querer ser; esto amerita en el odontólogo adquirir competencias gerenciales, que les permitan gestionar las expectativas y necesidades de los clientes- 

RELACIONAL.

pacientes como un activo intangible relacional, basado en los juicios y valores que se obtienen sobre la marca, identidad e imagen corporativa del profesional u organización odontológica a través de otros clientes, competidores, empleados potenciales, grupos de interés, familiares, entre otros; de manera que, la reputación corporativa contribuirá no solo en la creación de valor de la empresa, sino además, potenciará los niveles de satisfacción esperados sobre este activo en particular. Puesto que, al entender las necesidades de los clientes-pacientes mediante la retroalimentación sistemática, contribuirá a la gestión de una relación mutua de trabajo a largo plazo y, al mismo tiempo, potencializará la lealtad; y su constante monitoreo debe ser fundamental en cualquier planeación estratégica de la organización odontológica.

Para mejorar la retroalimentación o flujo de información sobre el profesional u organización odontológica con base en los elementos del capital relacional, se sugiere el uso de estrategias de dinamización que los acerquen tanto al entendimiento como las interpretaciones que el cliente-paciente posee respecto a la identidad que pretende alcanzar a través del servicio que busca contratar. Podemos mencionar en este caso, y en vista a la pandemia Covid-19, que se ha generado en la odontología el uso de herramientas digitales como la teleodontología que no son sustitutos de la consulta presencial, sino, un gestor que ofrece nuevas oportunidades para el acceso fácil a consulta, programa de educación y educación dental continua.

En suma, la intención de esta investigación fue confluir en el desarrollo de nuevas dimensiones del negocio odontológico a través de un mayor acercamiento a los clientes-pacientes (Figura 1).

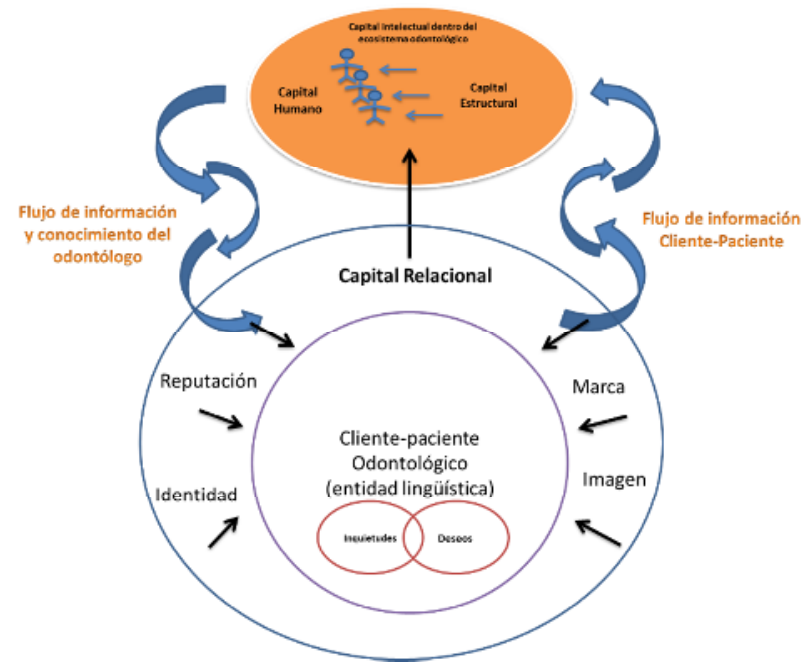

Figura 1. Identificación del capital relacional dentro la organización odontológica.

Fuente: Elaboración propia con base en Bontis (1998) los subdominios del capital intelectual dentro de las organizaciones empresariales. 

RELACIONAL.

En definitiva, el capital relacional del ecosistema odontológico (Figura 1) debe ser gestionado por el odontólogo como gerente de la organización, puesto que, la activación de los elementos que lo componen (la marca, identidad, la reputación e imagen corporativa del profesional) mediante la retroalimentación o flujo de información con los clientes-pacientes para potenciar el servicio y así poder alcanzar el mejoramiento de la sonrisa con base en la belleza facial y dental.

\section{Glosario de términos}

- Armonización Orofacial: "Un conjunto de procedimientos estéticos que, cuando se combinan, mejoran la simetría del rostro, transforman algunas características y tratan el envejecimiento de la piel, caracterizado por la pérdida de elasticidad, volumen y grasa previo a un diagnóstico clínico, es un nuevo concepto que busca destacar la belleza natural de cada persona".

- Cavidad Oral: "Se refiere a la boca que consta de los labios, el revestimiento interno de las mejillas y los labios, las dos terceras partes delanteras de la lengua, las encías superiores e inferiores, el piso de la boca (debajo de la lengua), el paladar duro (techo de la boca formado por hueso) y el espacio pequeño detrás de las muelas del juicio".

- Capital relacional: "Es el conjunto de conocimientos basado en las relaciones institucionales que mantiene una empresa con otros agentes, que le reporta un valor y una base de conocimientos necesarios para realizar su actividad de manera más eficiente".

- Clientes-pacientes odontológico: "Persona o individuo que acude a la organización odontológica cuando tienen dolor o molestia para sanar y por la otra, con el deseo de alcanzar la identidad individual o colectiva que desea con respecto a su sonrisa".

- Entidad lingüística: "Es el ser humano que provee de sentido su forma de vida fundamentado en reconfiguraciones lingüísticas que generan los modos de actuar".

- Familia: "Constituida por familiares y amigos, que brinda apoyo como mano de obra en la organización, lo cual hace un ahorro significativo en el gasto personal al comienzo de los primeros años de vida de la organización".

- Identidad e imagen corporativa: "Es la suma de los elementos tangibles e intangibles que distinguen a una organización que se proyecta con el objetivo de generar una impresión (imagen) especifica en los stakeholders con los que se relaciona la empresa". 

RELACIONAL.

- Marca: "Es el símbolo para la identificación, toma de decisiones de consumo e intercambio".

- Relaciones informales: "Representan las interacciones entre las personas en un contexto de cercanía, de amistad o de sintonía fraccionando las barreras estructurales organizativas".

- Reputación: "Es como un activo de construcción social que es instaurada y sostenida por los procesos de legitimación".

\section{REFERENCIAS BIBLIOGRÁFICAS}

Aafjes, M. (2008). Belleza producida y cuerpos maleables: un estudio sobre la belleza física y la práctica de cirugía estética en Buenos Aires. Tesis de Maestría. . Buenos Aires: FLACSO. Sede Académica Argentina.

Almazrooaa, S. A., Mansour, G. A., Alhamed, S. A., Ali, S. A., Akeel, S. K., Alhindi, N. A. \& Binmadi, N. O. (2021). The application of teledentistry for Saudi patients' care: A national survey study. Journal of Dental Sciences, 16(1), 280-286.

Aramburu, N., Sáenz, J. \& Blanco, C. (2015). Structural capital, innovation capability, and company performance in technology-based colombian firms. Cuadernos de Gestion, 15(1), 39-60. doi: http://doi.org/10.5295/cdg.130427na

Araujo, M. M. \& Morón, A. M. (2021). La Teleodontología una Herramienta Fundamental en Tiempos de Pandemia y post COVID-19, su Utilidad en las Diferentes Especialidades Odontológicas. Int. J. Odontostomat, 15(1), 43-50.

Arias, F. (2018). Editorial: Diferencia entre teoría, aproximación teórica, constructo y modelo teórico. Revista Actividad Física y Ciencias, 10(2), 7-12.

Association American Teledentistry, A. (2019). Teleorthodontics and Clear Aligner Treatment: An American Teledentistry Association Position Paper. Recuperado el 2 de 07 de 2021, de http://www.americanteledentistry.org/wpcontent/uploads/2019/12/ATDA-POSITIONPAPER_Finalcopy1.pdf

Atkin, D. (2008). El secreto de las marcas: clubes para gente muy especial . Barcelona: Robinbook. 

RELACIONAL.

Balmer, J. (2001). Corporate Identity, Corporate Branding and Corporate Marketing: Seeingthrough the fog. European Journal of Marketing, 35(3/4), 248-291.

Barquero, J. D. (2008). El libro de oro de las relaciones públicas y el marketing \& 34; think tank\& 34; en acción para mantener y obtener nuevos clientes . Grupo planeta (GBS).

Bauman, Z. (2007). Vida de consumo. México: Fondo de Cultura Económica.

Beltrán, R. J. (2013). Historia y Filosofía de la Odontología. Revista Estomatológica Herediana, 23(3), 167-167.

Bontis, N. (1996). "There is a price on your head: managing intellectual capital strategically". Business Quaterly , 41-47.

Bontis, N. (1998). "Intellectual capital: An exploratory study that develops measures and models". Management Decision, 36(2), 63-76.

Bontis, N. (1999). Managing organizational knowledge by diagnosing intellectual capital:framing and advancing the state of the field. International Journal of Technology Management, 18(5-8), 433-462.

Bontis, N. (2001). Assesing knowledge assets: a review of the models used to measure intellectual capital. International journal of management reviews, 3(1), 41-60.

Bontis, N. (2001). Assessing knowledge assets: A review of the models used to measure intellectual capital. International Journal of Management Reviews, 3(1), 41-60.

Bontis, N. \& Girardi, J. (2000). Teaching knowledge management and intellectual capital lessons: an empirical examination of the TANGO simulation. International Journal of Technology Management, 27(5-8), 545-555.

Bontis, N., Chua, C. K. \& Richardson, S. (2000). Intellectual capital and business performance in Malaysian industries. Journal of intellectual.

Bontis, N., Crossan, M. \& Hulland, J. (2002). Gestionar un sistema de aprendizaje organizacional alineando stocks y flujos. Revista de estudios de gestion, 39(4), 437-469.

Boone, L. \& Kurtz, D. (2012). Marketing Contemporáneo. México D.F.: Cengage Learning.

Borrás-Atiénzar, F., \& Campos-Chaurero, L. (2018). El capital intelectual en las empresas cubanas . Ingeniería Industrial, 39(1), 56-66.

Brûderl, J. \& Preisendôrfer, P. (1998). Network support and the sucess of newly founded business. Small business economics, 10(3), 213-225.

Bueno, E. (1998). Medición del capital intelectual: Modelo Intelect Euroform. IUEE San Lorenzo de El Escorial. 

RELACIONAL.

Bueno, E., Del Real, H., Fernández, P., Longo, M., Merino, C. \& Murcia, C. (2011). Modelo Intellectus de medición, gestión e información del capital intelectual. Madrid,España: Universidad Autónoma de Madrid.

Bueno, E., Salmador, M. \& Merino, C. (2008). Genesis, concepto y desarrollo del capital intelectual en la economia del conocimiento: una reflexion sobre el Modelo Intellectus y sus aplicaciones. Estudios de Economia Aplicada, 26(2), 43-64.

Carrascosa, J. (2021). La importancia de los lazos sociales: clases sociales y mecanismos de acceso al empleo en el Área Metropolitana de Buenos Aires. Estudios Sociologicos, $39(115)$

Cepeda-Palacio, S. (2014). Alcances actuales del concepto de marca. Un estudio comparativo, en la historia. Entramado, 10(2).

Chajet, C. \& Shachtman, T. (1998). Image by Desing: from Corporate Vision to Business Reality . Nueva York: McGraw-Hill.

Chiavenato, I. (2011). Administraciçon de recursos humanos. El capital humano de las organizaciones. McGraw-Hill.

Christensen, L. \& Askegaard, S. (2001). Corporate Identity and Corporate Image Revisited: a Semiotic Perspective. European Journal of Marketing, 35(3/4), 292-315.

Christie, D. (2002). Tesis Doctoral, School of Management, Faculty of Commerce and Management Griffith University. A Trilateral Model for the Management of Corporate Image: an Examination of the Inter-relationship between an Organization's self Image, its Projected Image and its Perceived Image.

Cortina, K., Flores, A. \& Álvarez, M. (2019). El capital estructural y su asociación estratégica con el desempeño organizacional: sector público y privado de Tamaulipas. VinculaTégica EFAN, 5(2), 1113-1126.

Costa, J. (1977). La imagen de la empresa. Métodos de comunicación integral. Madrid: IbéricoEuropa de Ediciones.

Currás, P. (2010). Identidad e imagen corporativas. Teoría y praxis, 9(34).

Damián-Navarro, L., Flores-Mori, M. \& Flores-Mena, B. (Ene-Mar de 2014). El consentimiento informado en Odontología, un Análisis Teórico. Revista Estomatológica Herediana, 24(1).

De La Hoz, E., López, L. \& Pérez, L. (2017). Modelo de gestión de relaciones con los clientes en empresas de consultoría. Investigación e Innovación en Ingenierias, 5(2), 46-77.

De la Hoz, G. E. (2017). Modelo de gestión de relaciones con los clientes en empresas de consultoría. Pdf Element, 5(2). 

RELACIONAL.

De Pablos, P. O. (2003). Intellectual capital reporting in Spain: a comparative view. Journal of intellectual capital .

Delgado-verde, M., Martin-de-Castro, G., Navas-López, J. \& Cruz- González, J. (2011). Capital social, capital relacionale innovación tecnológica. Una aplicación al sector manufacturero español de alta y media-alta tecnologia. Cuadernos de economía y Dirección de la Empresa, 14(4), 207-221.

Diaz, L. V. (2007). Gestión del conocimiento y del capital intelectual: una forma de migrar hacia empresas innovadoras, productivas y competitivas. Revista Escuela de Administración de Negocios, (61), 39-67.

Dowling, G. (1986). Managing your Corporate Images. Industrial Marketing Management, 15, 109-15.

Duque, O. \& Carvajal, P. L. (2015). La identidad organizacional y su influencia en la imagen: una reflexión teórica. Suma de Negocios, 6(13), 114-123.

Echeverría, R. (2000). La empresa emergente. La confianza y los desafíos de la transformación (1ra. 9na reimpresión 2012 ed.). Buenos Aires: Granica.

Echeverría, R. (2009). El observador y su mundo. Chile: Granica.

Edvinsson, L. \& Malone. (1997). "Developing intellectual capital at Skandia ". Long Range Planning, 30(3), 366-373.

Edvinsson, L. \& Sullivan, P. (1996). Desarrollar un modelo de gestión del capital intelectual. Revista europea de gestión, 14(4), 356-364.

Edvinsson, L. \& Malone, M. (1999). El capital intelectual. Barcelona: Editorial gestión 2000.

FDI, F. D. (1900). https://www.fdiworlddental.org/. Recuperado el 08 de 12 de 2020

Flores, A., Verástegui, J. \& Hernández, L. (2021). El capital estructural y relacional en el desempeño organizacional del sector público en el estado de Tamaulipas (México): Un análisis factorial exploratorio y correlacional usando Rho de Spearman. Acta Universitaria, 1-16. Obtenido de http://148.214.50.59/index.php/acta/article/view/2810

Flores-Flores, A., Bernal-González, I. \& Valentín-Mballa, L. (2020). Análisis bibliométrico del capital intelectual y desempeño organizacional. Ciencias Administrativas. Teoría y Praxis, 16(2), 123-142. doi:https://doi.org/10.46443/catyp.v16i2.265

Gotsi, M. \& Wilson, A. M. (2001). Reputación corporativa: buscando una definición. Comunicaciones corporativas: una revista internacional. 

RELACIONAL.

Gregnanin, I. \& Aulestia-Viera, P. (2017). Aplicación de la toxina botulínica para la armonización de la sonrisa gingival. Presentación de caso clínico y revisión bibliográfica Odontol. Sanmarquina, 20(2), 133-136.

Hatch, M. \& Schultz, M. (1997). Relations between organizational culture, identity and image. European Journal of Marketing, 31((5/6)), 356-365.

Heidegger, M. (1972). Ser y tiempo. (J. t. Rivera, Trad.) Santiago, Chile: Universitaria.

Hernández, R., Fernández, C. \& y Baptista, P. (2003). Metodología de la investigación (3ª ed.). México: McGraw-Hill.

Hsu, Y. H. \& Fang, W. (2009). Capital intelectual y desempeño en el desarrollo de nuevos productos: el papel mediador de la capacidad de aprendizaje organizacional. Pronostico tecnologico y cambio social, 76, 664-677.

International Society of Aesthetic Plastic Surgery. (2019). Encuesta Internacional Anual sobre Procedimientos Estéticos/Cosméticos. Recuperado el 4 de Agosto de 2020, de https://www.isaps.org/wp-content/uploads/2019/12/ISAPS-Global-Survey-2018-PressRelease-Spanish.pdf

International Society of Aesthetic Plastic, I. (2020). Procedimientos No Quirúrgicos. Recuperado el 04 de Agosto de 2020, de International Society of Aesthetic Plastic Surgery - Espanol: https://www.isaps.org/es/procedimientos/procedimientos-no-quirurgicos/

Klein, N. (2001). No logo: El poder de las marcas. Buenos Aires: Paidós.

Kotler, P. (2000). Dirección de Marketing: Análisis, planificación, gestión y control. Madrid: Prentice Hall.

Leal, F. A. \& Hernández, M. Y. (2016). Evolución de la odontología. Oral, 17(55), 1418-1426.

Ley del Ejercicio de la Odontología. (10 de Agosto de 1970). Gaceta oficial №. 29.288.

Ley del Ejercicio de la Odontología, O. (1970). Recuperado el 28 de enero de 2021, de http://www.elcov.org/ley1.htm

Lorenzo, P. C. \& Moreira, A. (2009). La gestión de las Relaciones con los clientes y la empresa de alta rentabilidad de Galicia. Revista de Estudios Regionales, 223-246.

Maluf, F., Santos de Melo, N. \& Garrafa, V. (2014). La Bioética en la relación profesionalpaciente en odontología. Acta Odontólogica Venezolana, 52(2).

Mancera, N. G. (2021). Propedéutica y semiología en odontología (2 da ed.). Barcelona, España: Elsevier. 

RELACIONAL.

Marcó, F., Loguzzo, H. \& Fedi, J. (2016). Introducción a la gestión y administración en las organizaciones. Argentina: Universidad Nacional Arturo Jauretche.

Margulies, W. (1977). Make the most of your Corporate Identity. Harvard Business Review, 6672.

Márquez, J. R. (2020). Teleconsulta en la pandemia por coronavirus: desafíos para la telemedicina pos-COVID - 19. Revista Colombiana de Gastroenterología, 35, 5-16.

Martínez, M. (2006). Ciencia y arte en la metodología cualitativa. México, D.F.: Trillas.

Medina, C., Gómez, S., Martínez, C., Ramírez, P. \& Bermúdez, C. (2010). Parámetros estéticos de la sonrisa aceptados por odontólogos especialistas y pacientes de ortodoncia. Revista Colombiana de Investigación en Odontología, 1(2), 228-237.

Michalisin, M. D., Kline, D. M. \& Smith, R. D. (2000). Activos estrategicos intangibles y desempeño de la empresa: un estudio multisectorial de la visión basada en recursos. Journal of Business Strategies, 17(2), 91-117.

Miranda, F., Chamorro, A. \& Rubio, S. (2007). Introducción a la gestión de la calidad . Madrid, España: Publicaciones Universitarias .

Núñez, I. (2000). Usos y definiciones de los términos relativos a los usuarios o clientes. Revista interamericana de bibliotecología, 23(1-2), 107-121.

Odontología, L. D. (1970). Recuperado el 28 de 01 de 2021, de https://www.elcov.org/ley1.htm Organización Mundial de la Salud \& OMS. $(7$ de abril de 1948). ttps://www.who.int/es/about/who-we-are.

Rao, H. (1994). The social construction of reputacion: Certificacion contest, legitimation, and the survival of organizations in the American automobile industry: 1895-1912. Strategic management journal, 15(S1), 29-44.

Rodriguez, F. J. (2004). La reputacion empresarial a partir de la perspectiva basada en los recursos y capacidades: una revisión teórica de su potencialidad competitiva. Investigaciones europeas de dirección y economía de la empresa, 10(2), 13-27.

Roos, G., Roos, J., Dragonetti, N. \& Edvinsson, L. (1997). Intellectual capital: Navigating in the new business landscape. NY: New York University Press.

Roos, J., Roos, G. \& Dragonetti, N. (2001). Capital Intelectual: el valor intangible de la empresa . Barcelona: Paidós.

Roos, J., Roos, G., Dragonetti, N. \& Edvisson, L. (1997). Capital intelectual: El valor intangible de la empresa.Editorial. (C. O. (2001), Trad.) Barcelona, España: Paidós. 

RELACIONAL.

Saenz, S. (2013). Perspectivas en la relación odontólogo-paciente: un vinculo entre personas. Facultad de Odontología UNcuyo, 7(2).

Saint-Onge. (1996). Tacit Knowledge: The key to the strategic alignment of intellectual capital. Strategy y Leadership, 24(2), 10-14.

Sala, A. M., De Cárdenas, S. O., Sala, A. A. \& González, J. J. (2000). Paciente... ¿Porqué? Revista cubana de estomatologia, 37(2), 123-126.

Sanchez, A., Melia, A. \& Garcia, J. (2007). El concepto del capital intelectual y sus dimensiones. Investigaciones Europeas de direccion y Economia de la Empresa, 13(2), 97-111.

Sanhueza, G. \& Cabrera, F. C. (2014). La evaluación de los aprendizajes orientada al desarrollo de competencias en Odontología. Revista Cubana de Educación Médica Superior, 28(1), 104-114.

Souza, C. G. \& et.al. (2021). Harmonização orofacial e a inter-relação com a implantodontia: Uma revisão de literatura. Research, Society and Development, 10(5).

Stewart, T. (1991). Brainpower: Intellectual Capital is Becoming Corporate America's most valable asset and cat be its sharpest competitive weapon, fortune, spring issue. 44-57.

Stewart, T. (1997). Intellectual capital: The new wealth of organizations. NY: Currency Doubleday.

Stewart, T. (1998). La nueva riqueza de las organizaciones: El Capital Intelectual. Buenos Aires: Gránica SA.

Stewart, T. (1999). Capital intelectual. Lisboa: Silabo.

Stewart, T. A. (1994). Intellectual capital: Your company's most valuable asset. Fortune vom, 3(94), 28-33.

Sveiby, K. (2000). Capital intelectual: La nueva riqueza de las naciones. Como medir y gestionar los activos intangibles para crear valor. (G. 2000, Ed.) Barcelona.

Terkla, D. \& Pagano, M. (1993). Understanding institutional image. Research in Higher Education, 34, 11-22.

Villafañe, J. (1998). Imagen positiva. Gestión estratégica de la imagen de las empresas. Madrid: Pirámide. 